\title{
Family members' involvement in the care of critically ill patients in two intensive care units in an acute hospital in Bahrain: The experiences and perspectives of family members' and nurses' - A qualitative study
}

\author{
Eman Ebrahim Fateel ${ }^{*}$ Catherine Sarah O'Neill \\ School of Nursing \& Midwifery, Royal College of Surgeons in Ireland, Bahrain
}

Received: September 22, 2015

Accepted: December 17, 2015 Online Published: December 30, 2015

DOI: $10.5430 /$ cns.v4n $1 \mathrm{p} 57$

URL: http://dx.doi.org/10.5430/cns.v4n1p57

\begin{abstract}
Objective: Family can play a vital role in promoting the overall well-being of the critically ill patient. Despite this positive attribute, some nurses, for various reasons are hesitant to include family in patient care. The study explored family members and nurses' perspectives and experiences of family involvement in caring for patients in intensive care units. The objectives were to describe both nurses and family members' perspectives and experiences of family involvement in care.

Methods: A descriptive exploratory qualitative study. Focus-group interviews were conducted using a purposeful sample of six family members of critically ill patients and six nurses from two intensive care units of a hospital in Bahrain.

Results: A central finding was that overall critical care nurses were reluctant to involve family in care, despite the fact that generally family members were willing to participate in care. Themes that were common to both participant groups were that caring practices and active communication were central to quality patient care. Both groups agreed that kinship roles and relationships were pivotal to caring practices and enabled a sense of security and reassurance for family and patients. Despite the fact that nurses were aware that family knowledge of the patient made it possible for them to personalize patient care, they were reluctant to have open communication with family members and to include them in care. Instead they favored policy development to guide family involvement.

Conclusions: Family members expressed the need to be part of caring practices. While the nurses acknowledged that family involvement in patient care benefited the patient they wanted policy guidelines to guide this participation in care. The findings suggest that role clarification of family members and clarification on the responsibilities of nurses are needed if family members are to be involved so that patient care adheres to international standards while maintaining cultural sensitivity to Islamic family values.
\end{abstract}

Key Words: Intensive care units, Family involvement, Family-centered care, Islamic family values, Nurses, Communication, Care guidelines

\section{INTRODUCTION}

The intensive care unit (ICU) is a complex clinical setting, where the focus is on the clinical needs of the patient ${ }^{[1]}$ with less attention given to family requirements. Clinical and technical proficiencies are viewed as an indispensable components for nurses working in the critical care, skills that

\footnotetext{
*Correspondence: Eman Ebrahim Fateel; Email: eefateel@rcsi-mub.com; Address: School of Nursing \& Midwifery, Royal College of Surgeons in Ireland, Bahrain.
} 
are frequently considered to be of greater importance than caring aspects. Critical illness often occurs without warning, leaving the critically ill relatives feeling vulnerable and helpless with no clear knowledge about the patient's prognosis or outcomes. ${ }^{[2-4]}$

\section{Background}

Family Systems Theory ${ }^{[5]}$ describes the family as an interdependent, interactive system whose whole is greater than the sum of its parts. In line with this perspective, Islamic family structures and values consider family relationships to be at the core of their system as opposed to societies where individuals and individual decision making takes precedence. Thus, in the Arab world maintaining family bonds and cohesiveness is an overall family goal and reflects decision making processes whereby Arab Muslims sacrifice their individuality for the sake of preserving family unity. ${ }^{[6,7]}$ Disturbance of the family system affects the unity and the dynamic relationship between family members, for example the admission of a family member to hospital with illness crises, can result in changes to the functioning of the entire family system. ${ }^{[8]}$ The impact of the admission of a family member to an ICU may be stressful to the support system resulting in a crisis within the family as it places the family beyond what is considered a normal sphere of coping. It has previously been established ${ }^{[2,9-11]}$ that meeting family members basic needs can facilitate effective coping mechanisms when dealing with the admission of a family member to the ICU. Some of these basic needs include proximity and being near the patient, information, reassurance and support. Studies ${ }^{[12-14]}$ suggest that a high level of family comfort may contribute to a largely favorable family experience when a family member is admitted to an ICU. It seems, however, that nurses frequently find it challenging to consider the care of the family as a vital component of the care of the patient, and often struggle to establish a balance between patient care and the care needs of family members. ${ }^{[8]}$

The interactional and interpersonal dimensions of helping situations are described as caring. ${ }^{[15]}$ Caring is a universal phenomenon in everyone's life, and a fundamental concept in relation to the nursing profession. ${ }^{[16]}$ In Islam, caring is considered a spiritual action, in which the caring individual receives rewards from God for good deeds. ${ }^{[17]}$ The spiritual dimension of caring for Islamic nurses positions caring as a fundamental part of patient care. Caring is a core concept of the Muslim faith and for Muslim nurses it defines and shapes their commitment to professional practice and their relationships with patients' with whom they share similar values thus, enhancing a sense of shared meaning within the professional caring relationship. ${ }^{[18]}$ Despite the difficulty of defining caring as a concept it is one that is accepted worldwide within the discipline of nursing. Some would argue that, nursing without caring, cannot be called nursing. ${ }^{[16]}$ It seems that caring practices are developed and enhanced with close interactions with patients and family members. ${ }^{[19]}$ Patients in ICUs in the main, because of their illnesses are unable to communicate their care needs, thus family members can play an essential role in communication and in acting for the "silent" patient. In Islamic culture, the extended family, especially immediate family members, play a key role in when a family member is sick. They receive and disseminate information related to the patient from the doctor and are very involved in the decision-making process. ${ }^{[20]}$ Currey and Worrall-Carter ${ }^{[21]}$ note that when nurses do not have social knowledge of the patient, and their family, clinical decision making and communication is more difficult. Patient-centeredness is an indicator of quality care, and the essence of a reciprocal relationship and inter-subjective experience that neither the patient nor healthcare providers can precisely define. ${ }^{[2]}$ Hoffman $^{[23]}$ and Al-Mutair et al. ${ }^{[24]}$ in two different cultural contexts studied the active involvement of families in ICUs and both study findings evidenced the advantages of increasing family involvement in the care of critically ill patients resulted in family centered care. Engstrom and Soderberg's ${ }^{[25]}$ focus group findings indicated that the presence of close family members was expected by nurses and it was frustrating for them and the patients if their family members were not proximally close. In many ICUs family members are not actively or consistently involved in patient care. ${ }^{[26,27]}$ Limited involvement in patient care activities challenges close family members to assume the likely expected role when the patient returns home. ${ }^{[23]}$ Additionally in the context of serious illness, individuals usually depend on others to help them think and feel their way through difficult decisions, as important health decisions are usually not made in isolation. ${ }^{[28]}$ Thus, the goal of this study was to explore family members' and nurses' perspectives and experiences of family involvement in caring for patients in ICU, with the objective of describing both nurses' and family members' perspectives and experiences of family involvement in care in order to gain an understanding of how the care process might be enhanced.

\section{Methods}

A qualitative research design using focus-group interviews was conducted to generate data. A semi-structured interview topic guide was developed to guide the focus group discussions, in order to achieve the overall study aim and objectives. Broad open questions were used only as guide (see Tables 1, 2), and the moderator used additional probes 
as necessary to stimulate and to focus the discussion. The interview topic guide was not significantly changed for either group of participants rather, the order of the questions were modified during the course of the interviews in order to refine and focus the study depending on the data being generated.
Two intensive care units of a large hospital in Bahrain were the study sites. The population were the family members of patients admitted to the ICUs and the critical care staff nurses working in the units.

Table 1. Family members interview guide

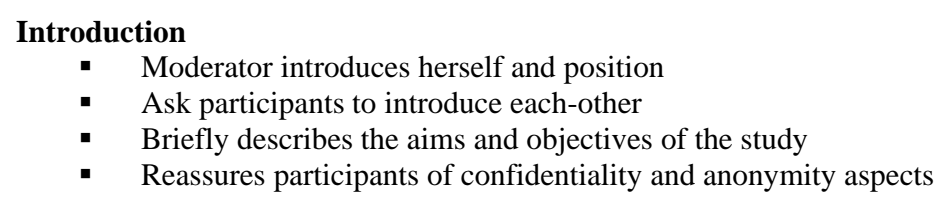

How do you feel about your (family members') involvement in the care of your loved ones (patients')? Guiding points/Probes

- Support/encourage this involvement

- Ability to share/engage in the care/decision making process

- Time given to address concerns and questions

- $\quad$ Obstacles encountered with/during your involvement

(If not) How do you think you could be involved? Would you like to be involved?

Tell me about how you received the information by nurses about your family member?

Guiding points/Probes

- Ease

- Understandable

- Completeness

- Consistency

Could you tell me what maybe be the benefits (if any) of your (families') involvement in the care of your loved ones (patients) are?

Guiding points/Probes

- Emotional needs

- Physical needs (critical/non-critical tasks)

- Spiritual needs

- Patient benefits

- Family members benefits

- Staff benefits

Would you tell me what the disadvantages (if any) of families' involvement in the care of their family members would be? Guiding points/Probes

- Patient safety

- Quality of care

What else would you like us to know so we can take better care of our patients and their families?

We would like to thank you very much for your participation and your opinions

The study used two focus groups; one consisted of the critical care nurses and the other of critical care patients' family members. A non-probability purposive sampling method was implemented with a sample of six participants from each group. Krueger and Casey ${ }^{[29]}$ suggested that a smaller group (between 6-8 participants) shows a greater potential to gain a variety of perspectives while also being small enough not to get disorderly or fragmented. Family members younger than 18 years unable to read, non-English speaking, and un- willing to participate or sign the consent form were excluded from the study. Only family members of non-trauma surgery patients who had been admitted to the ICUs for at least 48 hours and had experienced involvement in caring activities were approached to participate in the study. The criteria for participation for the critical care nurses' were that nurses should be registered with a valid clinical license, had worked for at least two years in intensive care settings and had experienced family members' involvements in caring activities. All 
participants, both family members and nurses were Muslims. per se the majority $(70.2 \%)^{[30]}$ of the population in Bahrain While the focus of the study was not on Muslim individuals are Muslims.

Table 2. Critical care nurses interview guide

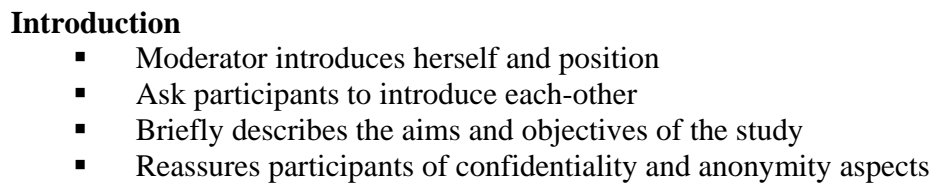

How do you feel about family members' involvement in the care of patient? Guiding points/Probes

- Support/encourage this involvement

- Ability to share/engage in the care/decision making process

- Time given to address family members concerns and questions

- Obstacles encountered with/during family members involvement

(If not) How do you think the family members could be involved? Would you like them to be involved? Tell me about how you have handed the information to the families' regarding their family member? Guiding points/Probes

- Ease

- Understandable

- Completeness

- Consistency

Could you tell me what maybe be the benefits (if any) of families' involvement in the care of their family members are Guiding points/Probes

- Emotional needs

- $\quad$ Physical needs (critical/non-critical tasks)

- $\quad$ Spiritual needs

- Patient benefits

- Family members benefits

- Staff benefits

Would you tell me what the disadvantages (if any) of families' involvement in the care of their family members would be? Guiding points/Probes

- Patient safety

- Quality of care

What else would you like us to know so we can take better care of our patients and their families?

We would like to thank you very much for your participation and your opinions

Prior to the commencement of the study the application was scrutinized by the Royal College of Surgeon in Ireland Medical University of Bahrain Research Ethics Committee (REC). Following approval from the REC the first author accessed the Ministry of Health REC. The first author then negotiated with the gate keepers in the Ministry hospital in order to gain access to the participants. Processes that ensured that attention was given to respecting all potential participants and also that the study was considered scientifically robust and that it was anticipated that the outcomes would be of beneficence to the wider community. Prior to the start of the study an information leaflet was displayed in the hospital inviting people to take part in the study. Those who were interested contacted the first author and she verbally outlined the study purpose to them prior to taking part; information leaflet about the general nature of the study, purpose, benefits and expectations was distributed as also informed consent forms. Informed consent was formally signed prior to start of the focus groups. The interviews took place in one of the ICU conference rooms. Group discussions were recorded using a Sony digital voice recorder and all the interview data was transcribed verbatim by the first author. Observational notes were made immediately after each interview by the assistant moderator and the first author to contribute to the accuracy of the data collected. First impressions and conclusions about the study process and outcomes were expressed during a de- 
briefing with the assistant moderator and the researcher after both focus group interviews. Participants' comments were integrated into a second draft report. Supplementary remarks were kept as additional information. All participants were allocated different codes to ensure confidentiality of data and anonymity of participants. The data was keep secure on a password encrypted computer. The only other person who read the data was the second author. The assistant moderator of the focus groups also signed a confidentially form. Details collected pertaining to patients and their hospitalization was taken from the participants' perspective and not from the patients' records. Participants had the right and the freedom to withdraw from the study at any time without any coercion of any type and they were reassured that their participation was voluntary.

\section{Data analysis}

Krueger's ${ }^{[31]}$ framework was used to analyze the data, which facilitated the emergence of themes. The framework consists of seven criteria for interpreting coded data including: words, context, internal consistency, frequency and extensiveness of comments, specificity of comments, intensity of comments, and big ideas. The purpose of choosing Krueger's framework analysis is not only to make sense of the participants' quotes, but also to be to see the relationship between the quotes and the links between the data. ${ }^{[32]}$ Through thematic content analysis; the process of analysis included a full transcription of the interview material, reading the material several times to gain a sense of the content. The whole transcript was then read again to identify, extract and classify data into condensed meaning units, bearing in mind the aim of the study. After selecting the units of significance or of meaning, units were sorted into categories. Those categories that were related to each other were subsumed into general themes. ${ }^{[29]}$ The categories of items and the reduction of items into themes were validated independently by the first author. Conceptually, similar events were grouped together to form a framework of categories and subcategories. Following discussion and comparison with the co-author, themes were revised and consensus was reached, that is significant for completeness of the interpretation and rigour. ${ }^{[33]}$

\section{Findings}

All respondents' were Bahraini, Muslims. Ages for both participant groups ranged between 26 to 38 years old. Nurses' participants work experience ranged from 2 to 13 years of experience, 2:4 male to female ratio respectively were recruited. Family members' participants all included close family members and were all females in gender. From the six family members, four members each were recruited from different patients and two family members from the same patient. All family members who participated in this study had no previous experience of caring practices in an ICU context, but some of them been exposed to an ICU environment. Data saturation was reached in both focus group interviews, as participants repeated information. All participants stated having experienced an open atmosphere, and been able to say what they wanted. Themes that were common to both participant groups were that caring practices and active communication were central to quality patient care (see Figure 1). Kinship roles and relationships were pivotal to caring practices and enabled a sense of security and reassurance for family and patients. Two main themes emerged from the analysis of family members' data: (1) caring aspects, and (2) communication and relationships. Two themes emerged from the analysis of the nurses' data: (1) family involvement, and (2) being involved.

\subsection{Family experiences}

\subsubsection{Caring aspects}

Being part of caring practices was important for family members. The subthemes that formed this core theme were: (1) desire to be involved, (2) the challenges of being there, and (3) the importance of support and respect.

\section{Desire to be involved}

Participants described how they wanted to be near their critically ill loved ones. Expressions of empathy and physical contact were interpreted by the family members as a sign of demonstrating care and support. Family members appreciated simple gestures of physical connection and even described that slight increase in physical closeness, like a touch of the hand, was therapeutic for both the family members and the patient during these stressful times, instilling a sense of security and reassurance:

"we have a common saying that the family should be always around the sick person, being there makes us feel better, because we can understand what is going on for my brother [the patient], if he is getting better or not and how we can help him" (AM).

"I feel it is [providing support] a step must be taken for the patient ... it is an element that helps the person to get back to his normal life" (FY).

Participants described how this need was linked to their kinship role and relationship to the patient and this made them feel it as a natural instinct and desire. While family members expressed the need to perform caring tasks for the patient, despite of the patient's condition, they expressed the need to 
have this care supervised by the respective nurse. Caring for the patient helped family members maintain family bonds and cohesiveness and helped to minimize the disturbance imposed on the family unit following a family member's admission to the ICU. Participants described how this caring was for them healthy and natural. They also seen it as a stepping stone enabling them to assist the patient in resuming their normal life when discharged. Participants when describ- ing this care referred to themselves as "long-term caregivers" compared to nurses which was described as a "temporary/ short-term caregivers": "we are going to continue the care that they give now, who is going to continue their job in the end?... us!!" (FY) "nurses should know that they give care for short time only... but family they will take care of him [the patient] for life" (AS).
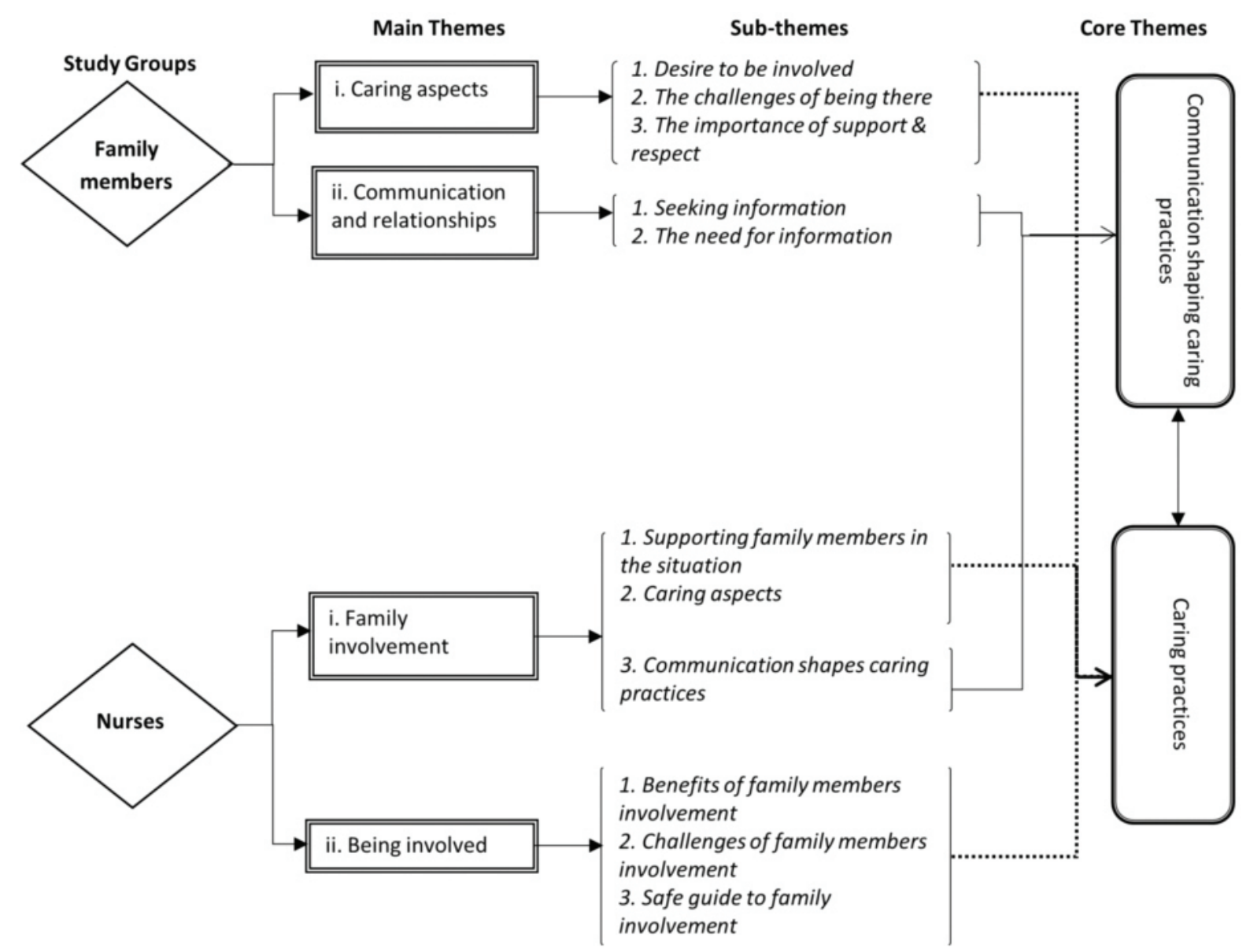

Figure 1. Common themes shared among family member's of critically ill patient \& critical care nurses' participant

\section{The challenges of being there}

An obstacle impeding their presence and the continuum of their natural role and relationship responsibilities were the restricted ICU visiting hours. Participants articulated the need for flexible visiting hours, and proposed that special consideration for unique access be given to at least one significant family member, based on kinship and relationship roles. Participants expressed concerns about the profeleration of visitors around their loved ones and suggested limiting visitors to maintain patient's safety and also give the patient a chance to rest: "not all family members should be around the sick person because I feel the patient is being harmed, my brother was harmed when he there are so many visitors and he still does!" (AM). "the nurse should explain that so many family members cause discomfort for the patient" (AS).

\section{The importance of support and respect}

Participants expressed the need for nurses to have a thorough consideration of the emotional impacts imposed on family members and to understand the family's need to be holistically supported, assessed on individual basis and to have more patience when dealing with family members during this critical time: "the nurse should give emotional support for the relative in spite of the patient condition, whether if she gives information or not, she should give emotional support 
for the relative" (AS). "nurses should support us [family] more, to be nicer and have more patience with us, as each one of us is different" (AY).

Participants expressed their wishes to be treated with dignity and respect. Most of the participants reported that they were ignored, and dealt with as if they did not exist:

"the nurses and the doctors they should treat the relative with humanity ... not to just explain to do the job and you don't feel for them! it's a part of their [nurses] job, a part of my right, to be treated like a human" (AS) "it's like we don't exist" (AM).

One of the participants described how a nurses was carless about the patient's confidentiality; they described how they had found the patient's diagnostic reports on the table. According to the participant, that the assigned nurse assumed that family members would not be able to read technical medical data:

"I found the CT report on my cousin's table... this report is confidential!!" (AS) "they [nurses] didn't consider that a family member could read it" (AS) "maybe we could or can read this report!" (FS).

\subsubsection{Communication and relationships}

Family members highlighted the need to establish a relationships based on effective communication skills with the nurses during the caring process. They described how such a relationship determined their participation in care. When communication was poor family members did not feel that they were being included in the care process. The two subthemes that constituted this theme were: (1) seeking information and (2) the need for information.

\section{Seeking information}

Family members expressed the view that that a good communicative relationship with the nurses was vitally important. Having full information enabled them to tailor care and more fully support their loved one. Unfortunately, many family members did not feel they had received full information about their family member: "no instructions or information provided, unless you keep on asking and asking ... my mother had to go after him [doctor] to ask him about my brother, as the nurse did not answer my mother questions clearly" (AY).

However, one participant voiced that some nurses were approachable and provided them with the needed support and information: "honestly when I asked the nurse she was so supportive, and gave me all the information in a simple way like explaining it for a baby, so easy to understand, although I didn't want that much of information, but she gave me anyway" (AM).

Many of the participants felt that the nurses were not being honest when asked or questioned about the patient's condition. They concluded that most nurses were afraid to disclose information related to patient's condition and were hesitant reveal if the patient's condition had deteriorated:

"other [nurses] were really, really hard to talk with and were unsupportive, so it depends on the nurse herself" (AY).

"she [the nurse] should give us information that is correct, but it doesn't mean that she should lie or hide something or say that she don't know ... she should explain that she is not allowed to give information and the doctor is responsible" (FS).

\section{The need for information}

Participants' elaborated further on how information could have enabled them to be more confident during the caring process and thus improve their emotional well-being, while reducing their worry and making them feel useful:

“if there wasn't enough information or instructions provided by the nurses, then we might hurt or harm the patient while caring" (AY).

"having information and being there can actually help us not only to give care but emotionally as well, better than being worried all the time about what will happen to him or if he is okay or not" (AY) "knowing what is going on makes us relaxed and feel better, so we do need it" (AY).

Family members' emphasized how they considered themselves as a rich source of information related to patient. They also elaborated how they could provide a comprehensive picture of the patient, such as his/her daily life, interests and habits, thus adding to the nurses' general knowledge of the patient: "the nurse has general information about the patient, and relative they are the other hand of patient" (AS).

\subsection{Nurses experiences}

\subsubsection{Family involvement}

The findings from the nurses' group discussion indicated several differences' of perspectives and experiences of family members' presence and involvement in care. The subthemes that emerged from the data analysis were: (1) supporting 
family members in the situation, (2) caring aspects, and (3) communication shapes caring practices.

\section{Supporting family members in the situation}

In the main, patients' admissions were unplanned, leaving family members emotionally unprepared for the illness event. The nurses in this study noticed that the ICU environment had a distinct impact on the family members and on their willingness to be involved in care. The more comfortable they felt in the ICU, the more they are able to support the patient. Some critical care nurses felt that it was important to prepare family members for the ICU environment, and to the critical illness of the patient especially if this was the first experiences of an ICU for family members. They considered that making family feel welcomed was an important part of the patient care and was vital towards initiating family participation in care: "we should encourage the relatives to involve.. we should welcome and encourage them [relatives] to come and talk with the patient ... with explanation they will involve in care" $(B)$.

\section{Caring aspects}

Most of the nurses favored to include only the immediate and closest family members in selected caring practices: "still the type of relative, maybe his wife feeding him, it is like acceptable for some patients but the common relative will be maybe very shameful for the patient that somebody is feeding him ... actually a wife or if he is child and the mother is feeding him it is helpful for him, maybe, even psychologically, relatives also they will feel that patient is taking feed from them, they will feel that he is little bit healthy ... so for both of them it will be beneficial" (MU).

However, one participant reasoned that involvement in care specially when attending to the personal hygienic needs of the patient, regardless the kinship and relationship roles to the patient, could endanger the patient privacy: "I don't like to ask the family to come inside the patient room and change the patient in front of them. I don't like that at all, not even just to show them how it is done ... I think there will be no privacy for the patient, after all he is still a patient even if they are his family" (MU).

In response to the above, participants voiced that nurses have the option to ask the family members to leave the patient room in situations where they feel that the integrity of patient is threatened.

\section{Communication shapes caring practices}

Providing information and using effective communication skills are central aspects of meeting family needs and in establishing an interactive reciprocal relationship. Although nurses discussed how, and when, families should be given information, it appears that, for whatever reason, it is not always practiced. Nurses reflected difficulties in communicating effectively with family members and some strongly acknowledged the significance of having a part in the communication process and supporting families: "if we explain it in an easy way, making it more simple, they will [get] involved in the feeding, but if we explain it in a difficult way and told them [family] that the patient is in the critical area, they will not [want to be] involved, but with our explanation and encouragement they will [get] involved" $(B)$.

One participant did not consider providing such information as of significance or value towards family members' involvement in care. Other participants, however, described how they could foster relationships through communicating with family members and asking them whether they wanted to participate in some of selected nursing care activities: "we should make the family aware about this [family role!] most of people [family] are not aware that they can involve in the patient care under the nurses supervision" $(R)$.

While most of the participants agreed it was important to be truthful and not withhold information when communicating with families about the patient's condition, others described situations where it was difficult to do so, especially if poor patient clinical outcomes were expected or if a physician had ordered them not to disclose any information to families or patients: "Actually we have limitation or restrictions in answering the family questions, because we are staff nurses, we are not doctors" (MA).

\subsubsection{Being involved}

The subthemes for the main theme of being involved were, (1) benefits, (2) and challenges of family members' involvement, and (3) safe guide to family involvement.

\section{Benefits of family members' involvement}

Most of the nurses described how family members were a rich source for information and gave them an inclusive view of the critically ill person. This information helped them to tailor patient-specific nursing care:

"we cannot give holistic nursing care without involving the family in the care ... we always think that we are the only ones who have to take decisions for the patient, but in many situations I was amazed how family members can bring options that can improve patient treatment and outcome we never thought of, they can open opportunities because we [nurses] are system oriented, we are disease oriented professionals, family thinks in a different way, family can al- 
ways try to integrate the social and emotional, physical and economical and a lot of aspects in regards to patient care, and once when we come to take a decision, they are a very good source, they can give you options sometimes you will never think of" (R).

Some nurses believed that including family members in selected minor caring activities, could enhance the patients overall well-being and provide moral support. This would benefit both the family members' and the patient while also assisting the nurses to give holistic nursing family orientated care:

"from my personal experience illnesses is like a heart shape, if you involve the loved ones [family] in care planning and delivering that care that could improve and strengthen the familiar bond between family and their loved ones, as long as that involvement is reasonably induced taking in consideration the patient benefit and preventing harm that can have a great influence and impact on the family relationship. 'Beneficence' means we have to do everything that is good to the patient, involving family in the care, is something good, we need to move our standard of care a little bit one step ahead, and not just to keep on delivering a very traditional nursing care" (R).

\section{Challenges of family members' involvement}

Although nurses acknowledged the benefits of including family in care many were hesitant to do so. It appeared that nurses previous experience, busy schedule, the patients clinical condition, type of caring activity, and family relationships for example, kinship role and emotional responses and awareness, were factors that influenced their willingness to include family members in care. For some of the nurses their previous experiences of family involvement were not positive. Some felt that family lacked the skills of care and respect toward the nurses' role. Also some participants felt uncomfortable and stressed to be constantly observed at the bedside by the visiting family and blamed if something goes wrong: "the family are like spectators watching the game over the days over the course of the illness ..." (R). "If you involve the family to do something for the patient and if any mistake happen they will blame the nurse" (A).

The nurses described how it was challenging for them to establish a balance between supporting the family members and providing care to the critically ill patient. They described this as being torn between caring for the patient as their priority, and recognizing their responsibility towards families:
"Obstacle, it is actually the time, teaching relatives and asking them to involve in care will take time especially we are here in ICU ... our time is limited and mostly the patient condition is very critical and we are afraid of giving anyone a task because it is our responsibility ... giving someone else any task, maybe it could harm the patient ..." (MU).

In addition to the busyness of the unit the participants regarded the ICU visitation hours to be insufficient and an obstacle towards the establishment of a partnership between the nurses and family members: “... here in ICU we have visiting hours only 4 to $5 \mathrm{pm}$ and 7 to $8 \mathrm{pm}$ so it is only two hours, and each person should stay 5 minutes only, so I don't think during this 5 minutes he can do one task even feeding will take more than 5 minutes...” (MA).

Family visitation and participation in caring activities, was from the nurses' perspectives very depended on the patients clinical condition and the type of treatment they were receiving. Care by a family member who is a non-healthcare professional raised concerns for the nurses about patient safety and quality of care and was expressed as a barrier to family presence and participation. As patients' situations are variable and most families tend to be emotionally distraught, nurses recommended that family presence should be an option and nurses suggested that family participation in care should be preceded by special training with nurses' supervision for close monitoring for quality of care:

"Even if it is a simple task, still it should be supervised by the nurse, you cannot leave them alone, patient might suffocate with the feed ... your existing will give self-confident to the relatives themselves and safety for the patient" (MU).

“... important things you have to take into consideration, whether the patient is connect to monitoring devices or infusions ... these things should be taken into consideration before allowing the family to be involved ... if the patient is not connected to any of these things monitoring devices or dangerous drug infusions, we can give the family the role to involve in care ..." (R).

Participants had concerns in involving family members in direct nursing tasks or critical tasks and they suggested that families could be involved in non-direct caring tasks, for example, emotional, and religious supports: "family should not be involved in every and each aspect of care ... they should handle only the task which does not require an expert intervention" $(R)$. 


\section{Safe guide to family involvement}

Each patient and patient's family member represent a unique situation that calls for an individualized approach to family presence and participation in care, and all of the nursing participants were in favor of having a "safe guide to family presence and involvement", a clear policy that would describe the extent of family members' involvement and their limitations during family visits and participation in care:

"... we want to have the clear rules about including patient relative in caring, like in positioning is it allowed in ICU to share? ... if it is allowed definitely I will ask him [a relative] to come with me ... I will ask the brother ... we want to have clear rule about that, is it allowed or not allowed? Is it possible with arterial line, central line?" (S).

"to regulate and to establish a procedure and a policy explaining to what extend the family should be involved in care, it should not be an open statement it should be regulated like any other procedure..." (R).

"... the rule should be clear for us, what the relative can do and what they cannot do? so if there is a clear rule about this or policy then we can promote it, but from beginning there is nothing, professionally there is nothing so we cannot even promote this partnership" (MA).

\section{DiscuSsion}

While the study did not intended to target on specific Muslim population, all the respondents however who consented and were willing to be part of the study were Muslims. The sample was a purposeful judgmental one and did not focus on the nature of the family relationships or gender. Rather the focus of the study was on family involvement in patient care as in Islamic culture family is central of everyday day life and care. Despite the fact that all of the family members who participated in this study had no previous experience of caring practices in an ICU context, all were very positive about being involved in care.

The findings revealed that, having an interactive relationship and being part of caring practices, improved family members overall well-being, self-esteem, role recognition, and reduced feelings of fears, helplessness, and guilt during the crisis, and also generated a sense of usefulness a finding that was consistent with other studies. ${ }^{[2,10,34,35]}$ However, a major constraint for families wishing to participate in care was the visiting restrictions imposed by the units' visitation policy. Families in this study interpreted these restrictions as being denied access to their loved ones. Islamic teachings, affirm and encourage family values and to maintain a bond of family cohesiveness. In Bahraini culture, this mutual commitment is reflected through regular family visits, providing support when needed especially during illness or disturbance to the family unity. This supportive link also extends to neighbours and other members of the community. Islam is a religion that is socially grounded in relationships with all members of a family and community experiencing a sense of social cohesion. This very significant in a Muslim community. Thus, restricting visitors may be challenging especially if the patient is dying as extended family and neighbours may want to visit to say farewell. Similarly, studies have found that flexible visiting hours helped to link and maintain familial bonds as family members reported feelings of helplessness and powerlessness when visiting times were restricted. ${ }^{[36,37]}$ Immediate family members had concerns about the patient safety and well-being when an overabundance of visitors resulted in the critically ill person not getting sufficient rest. All of the participants with the exception of two participant, would have liked the nurses to control the number of visitors around the patients. Some family members suggested restricting the visitors only to close members.

During the stressful times of ICU admission, family members need to be assured that their loved ones are receiving the best care possible. Communication plays a central role in this process. However as family members were not allowed to be around the patient during doctors' rounds, they were left with insufficient information. Without sufficient information they were not able to have a genuine appraisal of the patient's condition, a finding that is congruent with other studies. ${ }^{[10,27]}$ Having a picture of what is happening to their loved ones, offers a pathway on how to provide safe and effective care and helps in minimizing distress. Family members described how they sometimes blamed the nurses for not providing enough and or clear information about the patient's condition and they were critical, stressed and sometimes aggressive towards the nurses when their information needs were not met. ${ }^{[8,38]}$ The majority of families wanted to receive honest and straightforward information and they found it frustrating receiving different versions of information from different nurses. They acknowledged that most nurses were hesitant and or afraid to disclose any patient details when asked. This left the family feeling anxious, frustrated and waiting longer times to obtain the information needed from physicians; they felt as if they were chasing around for information. In times of crisis often people do not have the emotional capacity to seek information, and several family members made the comment that they sometimes tended to be persistent when seeking information and 
unintentionally irritating the nurses when their needs were being ignored. Family members agreed that it was important to establish a mutual relationship based on effective communication, shared understanding, honesty, trust, respect and support. To reach a deeper understanding between participants in a dialogue, participants should address each other equally, avoid using power or restricting contribution, and most importantly respecting the personal values and decisions of each other's. ${ }^{[39]}$ Family members highlighted their need for emotional support and to be acknowledged and assessed on individual basis. ${ }^{[37]}$ Some family members mentioned that nurses were supportive and helpful while others described how they experienced the dignity of the patient being violated. The example of a nurse commenting on the smell of a patient was cited and this triggered strong emotional reaction and summoned them to defend and protect their loved ones.

Most of the nurses in this study acknowledged the significance of active communication, sharing information and supporting family in a caring manner and meeting their essential needs. Some nurses thought it would be better to ask the family members whether they wanted to participate in care or not. Nurses often forgot to ask and most of the family members were unaware that they could be involved. While the majority of nurses agreed on the importance of being truthful about how critical the patient might be, they also described situations where it was challenging to reveal the seriousness of the patient condition. For example, when a physician ordered not to disclose any information to the patient and or the patient's family. Mok and Chiu's ${ }^{[40]}$ study showed that nurses who had developed relationships based on trust with families and patients, had a holistic view on patient care, and while, the nurses in this study were somewhat supportive of fulfilling the family's needs for information they were less supportive of family involvement in care. The nurses were aware of how different family members could be. Some families questioned everything while others felt safe with the care provided. Despite the above, one nurse expressed the view that family reactions were a healthy phenomenon, regardless of the unintentional and undesirable impact on nurses. Overall the nurses in this study agreed that families are a valuable source of information and helped nurses give individualized patient care. ${ }^{[27]}$

The nurses generally believed that when family members are given the opportunity to assist in selected non-critical caring tasks that this was of benefit to both groups. ${ }^{[27]}$ The benefits of such involvement, enabled the nurses to focus on the complicated technical tasks of care. As the majority of patients in the ICUs were intubated and unable to actively communicate with the nurses, involving family members in care meant that the nurses were able to gain an understanding and knowledge of the patient through the family. According to nurses in this study, families' role and relationship to the patient and their previous exposures to such settings shaped the extent of family members' involvement and interactions in care. If the nurses considered that family members were close to the patient, they were more inclined to include them in care. On the other hand, while some nurses expressed a wish to interact with families, and wanted them to be involved in care they were concerned with patient safety. ${ }^{[12]}$ The nurses in this study suggested that family participation in care should probably be preceded by a special training along with nurses' supervision for close monitoring for quality of care. The nurses also emphasized that family presence should be an option as patients' situation are variable and most families at this time tend to be emotionally distraught. An additional consideration was that the nurses found it uncomfortable and stressful to be constantly observed at the bedside by the visiting family members, who they perceived as finding fault with their work.

All nurses, however, requested a clear policy to guide the extent of family involvement and to ensure that standards of care were consistently adhered to. The nurse were also concerned that an individualized approach should be taken to family involvement, one that ensured patient safety and quality of care to achieve what Potter and Mueller ${ }^{[41]}$ described as "knowing your patient". Lovering's ${ }^{[42]}$ crescent model of care may help critical care nurses, particularly in an Islamic culture to better know the patient and patient's spherical social network. This knowledge may contribute to a more individualized level of care that is respectful and sensitive to Islamic patients and encompasses the family values and family needs. Nurses in this study described how higher levels of communication are needed to foster a partnership in care with families. This was challenging as the ICU visiting hours were insufficient and ultimately could be considered an obstacle towards the establishment of such partnership.

\section{Conclusion}

Themes that were common to both participant groups were that caring practices and active communication were central to quality patient care. The findings in this study reflected positive and negative experiences of both families and nurses towards family interacting with their loved ones in the ICU. While nurses differed in their experiences and opinions about family presence, the findings showed that, patients' families felt differently. Overall, in this study there was a mismatch between the critical care nurses who were reluctant to involve family in care and the family members who were willing to take part in care. The findings indicate that there are many 
challenges to family involvement in patient care. Unresolved issues identified in this study include, inadequate visitation policy, poor communication, and the concept of family support and unguided involvement. The findings highlight the frustration felt by many family members while trying to be present for their loved ones, to help out and to maintain the social family unity, structure and function. This is an aspect of care that is particularly relevant in an Islamic culture. Since majority of the family members in the study expressed willingness to take part in caring practices of their loved ones, including family members in care should be a goal per se. The nurses found that they could provide focused individualized patient care when family was actively involved in care. Family members were considered by the nurses as good source of information, despite the fact that a culture of blame was imposed on nurses when patient care and recovery did not seem to progress. The nurses wanted family involvement in care to be guided by policy that would shape the degrees of family involvement within the ICU context to ensure the delivery of quality care and patient safety. This lack of clarity illustrates the need for developing protocols that would guide such inclusion in care. While this study has identified many positive aspects of families' inclusion for both nurses and families, it has also illustrated areas where such inclusion requires further support if to be established.

\subsection{Limitations}

The inclusion of the participants from the researcher's previous work unit could be seen as a bias and a limitation to the study. However, the researcher's work role was changed before the commencement of the study. Including two family members from the same patient could indirectly create bias or influence members' way of expressing feelings or opin- ions. In addition, the nature of the family relationship and characteristics were not examined in this study.

\subsection{Relevance to clinical practice}

The findings from this study indicated that involving the family members in care helped them to feel involved and appeared for some to reduce the stress associated with having an ill relative in the ICU. Involving family members in care can help critical care nurses plan interventions that focus on involving family members in patient care. Professional practice could benefit from nurses having a better understanding of the needs of family members, regardless of how demanding the nursing care might be. Acknowledging and meeting family needs in a human caring way is as important as carrying out task-oriented nursing care.

Recommendations from the study evidence that role clarification and the responsibilities of nurses and families are needed to ensure that patient care adheres to international standards and is also sensitive to Islamic family values to ensure that family centered-care is practiced. The findings provide valuable insights and offer recommendations for the development of family-centered care, family support sessions or support groups and educational programmes. Future research should focus on meeting family needs.

\section{ACKNOWLEDGEMENTS}

This study was carried out and submitted in part fulfillment of the degree of MSc Nursing in the School of Nursing \& Midwifery, Royal College of Surgeon in Ireland - Medical University of Bahrain, 2013.

\section{CONFLiCTS OF INTEREST Disclosure}

The authors declare no competing interest.

\section{REFERENCES}

[1] Plakas S, Cant B, Taket A. The experiences of families of critically ill patients in Greece: A social constructionist grounded theory study. Intensive and Critical Care Nursing. 2009; 25: 10-20. http://dx.doi.org/10.1016/j.iccn.2008.04.003

[2] Davidson JE. Family Centred Care: Meeting the needs of patients' families and helping families adapt to critical illness. Critical Care Nurse. 2009; 29: 28-35. http://dx.doi.org. ezproxy.rcsi-m ub.com/10.4037/ccn2009611

[3] Linnarsson J, Bubini J, Pereius K. Review: a meta-synthesis of qualitative research into needs and experiences of significant others to critically ill or injured patients. Journal of Clinical Nursing. 2010; 19: 3102-3111. PMID:20738453. http://dx .doi .org/10.1111 /j.1365-2702.2010.03244.x

[4] Al Mutair A, Plummer V, O'Brien A, et al. Family needs and involvement in the intensive care unit: a literature review. Journal of Clinical Nursing. 2013; 22: 1805-1817. http://dx.doi.org/10.1111/j ocn. 12065

[5] Bowen M. Family Therapy in Clinical Practice. Northvale: Jason Aronson; 1985.

[6] Rassool GH, Sange C. Understanding the Muslim family system. In: Cultural Competence in Caring for Muslim Patients (Rassool GH ed.). Basingstoke: Palgrave Macmillan; 2014. 52-71 p.

[7] Lovering S. The Crescent of Care: a nursing model to guide the care of Arab Muslim patients. Diversity and Equality in Health and Care. 2012; 9: 171-178.

[8] Sacco TL, Stapleton MF, Ingersoll GL. Support Groups Facilitated by Families of Former Patients: Creating Family-Inclusive Critical Care Units. Critical Care Nurse. 2009; 29: 36-45. http: //dx.doi.org/10.4037/ccn2009265

[9] Omari F. Perceived and unmet needs of adult Jordanian family members of patients in ICUs. Journal of Nursing Scholarship. 2009; 41: 28-34. PMID:19335675. http://dx.doi.org/10.1111/j.1 547-5069.2009.01248. $x$ 
[10] Hinkle J, Fitzpatrick E, Oskrochi G. Identifying the perception of needs of family members visiting and nurses working in the Intensive Care Unit. Journal of Neuroscience Nursing. 2009; 41: 85-91. PMID:19361124. http://dx.doi.org/10.1097/JNN.0b013e3 $1819 \mathrm{c} 2 \mathrm{db} 4$

[11] Bailey J, Sabbagh M, Loiselle C, et al. Supporting families in the ICU: a descriptive correlational study of informational support anxiety, and satisfaction with care. Intensive \& Critical Care Nursing. 2010; 26: 114-122. PMID:20106664. http://dx.doi.org/10.10 16/j.iccn.2009.12.006

[12] Azoulay E, Pochard F, Chevret S, et al. Family participation in care to the critically ill: Opinions of families and staff. Intensive Care Medicine. 2003; 29: 1498-1504. http://dx.doi.org/10.1007 /s00134-003-1904-y

[13] Davidson JE, Powers K, Hedayat KM, et al. Clinical practice guidelines for support of the family in the patient-centered intensive care unit: American College of Critical Care Medicine Task Force 2004-2005. Critical Care Medicine. 2007; 35: 605-622. PMID:17205007. http://dx.doi.org/10.1097/01. CCM. 0000 254067.14607.EB

[14] Mitchell M, Chaboyer W, Burmeister E. Positive effects of nursing intervention on family-centred care in adult critical care. American Journal of Critical Care. 2009; 18: 543-552. http://dx. doi .org /10.4037/ajcc2009226

[15] Taylor SG, Renpenning K. Self-Care Sciences, Nursing Theory, and Evidence-Based Practice. New York: Springer Publishing Company; 2011. $101 \mathrm{p}$.

[16] Scheel ME. Interactional Nursing Practice (3rd ed.). Denmark: Munksgaard, Copenhagen; 2005.

[17] Lovering S. Caring as an act of spirituality: a nursing approach. In: Cultural Competence in Caring for Muslim Patients (Rassool GH ed.). Basingstoke: Palgrave Macmillan; 2014. 27-38 p.

[18] Lovering S. How universal are the caring models used by nurses? Diversity and Equality in Health and Care. 2012; 9: 167-170.

[19] Sundin K. Sense of understanding and being understood in the care of patients with communication difficulties. Umea University Medical Dissertations, New Series No 699: Umea, Sweden; 2001.

[20] Rassool GH. Putting cultural competence all together: some considerations in caring for Muslim patients. In: Cultural Competence in Caring for Muslim Patients (Rassool GH ed.). Basingstoke: Palgrave Macmillan; 2014. 272-300 p.

[21] Currey J, Worrall-Carter L. Making decisions: Nursing practices in critical care. Australian Critical Care. 2001; 14: 127-131. http: //dx.doi.org/10.1016/S1036-7314(01)80030-8

[22] McAdam JL, Arai S, Puntillo KA. Unrecognized contributions of families in the intensive care unit. Intensive Care Medicine. 2008; 34: 1097-1101. PMID:18369593. http://dx.doi.org/10.1007 /s00134-008-1066-z

[23] Hoffman LA. Active Involvement of Families in ICU Care Improved Satisfaction with Care Provided. American Journal of Critical Care. 2009; 18: 543-552. http://search.proquest. com.ezproxy. rcsi-mub.com/docview/762276352?accountid=51580

[24] Al Mutair A, Pulmmer V, Clerehan R, et al. Needs and experiences of intensive care patients' families: a Saudi qualitative study. British Association of Critical Nurses. 2014; 19: 135-144. http://dx.doi.org/10.1111/nicc. 12040

[25] Engstrom A, Soderberg S. Close relatives in intensive care from the perspective of critical care nurses. Journal of Clinical Nursing. 2007; 16: 1651-1659. http://dx.doi.org/10.1111/j.1365-2 $702.2005 .01520 . x$
[26] Hughes F, Bryan K, Robbins I. Relatives' experiences of critical care. Nursing in Critical Care. 2005; 10(1): 23-30. PMID:15739636. http://dx.doi.org/10.1111/j.1362-1017.2005.00091.x

[27] Agard AS, Maindal HT. Interacting with relatives in intensive care unit: Nurses' perceptions of a challenging task. Nursing in Critical Care. 2009; 14: 264-272. http://dx.doi.org/10.1111/j.147 8-5153.2009.00347. $\mathrm{x}$

[28] Epstein RM, Street RL Jr. The values and value of patient-centered care. Annals of Family Medicine. 2011; 9: 100-103. http://dx.d oi.org/10.1370/afm.1239

[29] Krueger RA, Casey MA. Focus Groups: A Practical Guide for Applied Research (3rd ed.) CA: Thousand Oaks, Sage Publications; 2000. http://dx.doi.org/10.1037/10518-189

[30] Ministry of Information Affairs. Population and demographics. 2014 Available from: http://www.mia.gov.bh/en/Kingdom-of-B ahrain/Pages/Population-and-Demographic-Growth.as px (accessedon22June2015)

[31] Krueger RA. Focus Groups: A Practical Guide for Applied Research. Thousand Oaks, Sage Publications, CA; 1994.

[32] Rabiee F. Focus-group interview and data analysis. Nutrition Society. 2004; 63: 655-660. http://dx.doi.org/10.1079/PNS2004399

[33] Yardley L. Dilemmas in Qualitative Health Research. Psychology and Health. 2000; 15: 215-228. http://dx.doi.org/10.1080/0 8870440008400302

[34] Wahlin I, Ek A, Idvall E. Empowerment from the perspective of next of kin in intensive. Journal of Clinical Nursing. 2009; 18: 2580-2587. PMID:19538566. http://dx.doi.org/10.1111/j.1365-2702. $2008.02744 . x$

[35] Karlsson C, Tisell A, Engstrom A, et al. Family members' satisfaction with critical care: A pilot study. Nursing in Critical Care. 2011; 16: 11-18. PMID:21199550. http://dx.doi.org/10.1111/j.1 478-5153.2010.00388.x

[36] Kynoch K, Chang AM, Coyer F. The effectiveness of interventions to meet family needs of critically ill patients in an adult intensive care unit: A systematic review. JBI Library of Systematic Reviews. 2011; 9: 2829-2874.

[37] Verhaeghe S, Defloor T, Van Zuuren F, et al. The needs and experiences of family members of adult patients in an intensive care unit: A review of the literature. Journal of Clinical Nursing. 2005; 14: 501-509. PMID:15807758. http://dx.doi.org/10.1111/j $.1365-2702.2004 .01081 . x$

[38] Jacobowski NL, Girard TD, Mulder JA, et al. Communication in critical care: Family rounds in the intensive care. American Journal of Critical Care. 2010; 19: 421-430. http://dx.doi.org/10.40 $37 /$ ajcc2010656

[39] Walseth LT, Schei E. Effecting change through dialogue: Habermas' theory of communicative action as a tool in medical lifestyle interventions. Medicine, Health Care, and Philosophy. 2011; 14: 81-90. http://dx.doi.org/10.1007/s11019-010-9260-5

[40] Mok E, Chiu P. Nurse-patient relationships in palliative care. Journal of Advanced Nursing. 2004; 48: 475-483. http://dx.doi.org/1 $0.1111 / \mathrm{j} .1365-2648.2004 .03230 . \mathrm{x}$

[41] Potter P, Mueller JR. How well do you know your patients? Nursing Management. 2007; 38: 40-48. http://dx.doi.org/10.1097/0 0006247-200702000-00012

[42] Lovering S. Arab Muslim nurses' experiences of the meaning of caring. Unpublished DHSc thesis. Sydney, Australia: Faculty of Health Sciences, University of Sydney. 2008. http://hdl . handle.net /2123/3764 